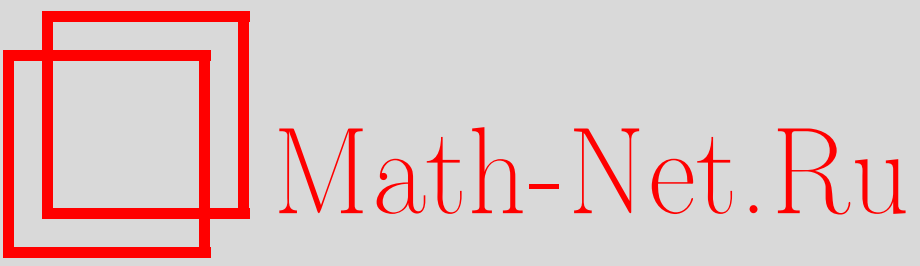

Я. В. Невмержицкий, А. В. Конюхов, Метод линий тока для расчета композиционной неизотермической фильтрации вязкопластичных нефтей, Матем. моделирование, 2020, том 32, номер 4, 75-93

DOI: https://doi.org/10.20948/mm-2020-04-06

Использование Общероссийского математического портала Math-Net.Ru подразумевает, что вы прочитали и согласны с пользовательским соглашением

http://www.mathnet.ru/rus/agreement

Параметры загрузки:

IP: 52.6 .47 .48

26 апреля 2023 г., $16: 46: 12$ 


\title{
МЕТОД ЛИНИЙ ТОКА ДЛЯ РАСЧЕТА КОМПОЗИЦИОННОЙ НЕИЗОТЕРМИЧЕСКОЙ ФИЛЬТРАЦИИ ВЯЗКОПЛАСТИЧНЫХ НЕФТЕЙ
}

\author{
(C) 2020 2. $\quad$ Я.В. Невмержицкий ${ }^{1}$, А.В. Конюхов \\ ${ }^{1}$ Московский физико-технический институт \\ 2 Объединенный институт высоких температур РАН \\ nevmerzhitski_y@mail.ru, konyukhov_av@mail.ru
}

DOI: $10.20948 / \mathrm{mm}-2020-04-06$

Целью работы является разработка численного метода, позволяющего проводить расчеты неизотермической многокомпонентной фильтрации за более короткое время, чем существующие конечно-объемные методы. Рассматривается плоская задача фильтрации воды, нефти и газа. Нефтяная фаза состоит из двух компонентов легких и тяжелых фракций, которые, как и вода, могут переходить в газовую фазу. В работе учтена не только нелинейность закона фильтрации нефти, но и зависимость параметров этого закона от температуры. Такая постановка задачи актуальна при моделировании разработки месторождений высоковязких нефтей. Для уменьшения вычислительной сложности задачи применен метод линий тока с расщеплением по физическим процессам, заключающийся в отделении конвективного переноса, направленного вдоль распространения потока, от процессов, связанных с теплопроводностью и гравитацией, направление которых не совпадает с конвективным потоком. Отличительной особенностью предлагаемого метода является совместное решение уравнений для давления, баланса энергии и массы компонентов как на линиях тока, так и на исходной сетке. Эта особенность позволяет проводить корректные расчеты при фильтрации нефтей со сложной реологией, зависящей от температуры. Численное решение системы уравнений фильтрации на двумерной сетке и линиях тока осуществляется методом IMРЕС. Для представленного метода линий тока предложен алгоритм учета теплопроводности, а также критерии перехода между расчетами на линиях тока и на двумерной сетке. Разработанная программа была верифицирована путем сравнения с аналитическими решениями, а также с результатами расчетов конечно-объемными методами на пятиточечном и девятиточечном разностных шаблонах.

Ключевые слова: высоковязкие нефти, нелинейная фильтрация, композиционное моделирование, метод линий тока.

STREAMLINE METHOD FOR SIMULATION

OF COMPOSITIONAL NONISOTHERMAL FLOW OF VISCOPLASTIC OILS

Y.V. Nevmerzhitskiy ${ }^{1}$, A.V. Konyukhov ${ }^{2}$ 
${ }^{1}$ Moscow Institute of Physics and Technology

${ }^{2}$ Joint Institute for High Temperatures of the RAS

The purpose of this work is to develop a numerical method that allows to carry out calculations of nonisothermal compositional flow faster than traditional finite-volume methods. A plane problem of oil, water and gas flow is considered. Oil phase is represented by two components - light and heavy fractions, which like water, can gasify. The work takes into account not only the nonlinearity of the oil flow law, but also the temperature dependence of parameters of this law. This statement of the problem is relevant for modeling of high-viscosity oilfields development. To reduce the computational complexity of the problem, the streamline method with splitting by physical processes is used, which consists in separating convective transport directed along the flow propagation from processes associated with heat conduction and gravity, the direction of which does not coincide with the convective flow. A distinctive feature of the proposed method is the joint solution of pressure equations, energy and components mass balance both on streamlines and on the initial grid. This feature allows to perform correct calculations for oil flow with complex temperature-dependent rheology. Numerical solution of system of flow equations on two-dimensional grid and on streamlines is performed by IMPEC method. For the presented streamline method, an algorithm for taking into account thermal conductivity, as well as transition criteria between calculations on streamlines and on a two-dimensional grid is proposed. The developed program was verified by comparison with analytical solutions, as well as with the results of calculations by finite-volume methods on five-point and nine-point difference stencils.

Key words: viscoplastic oils, nonlinear flow, compositional simulation, streamline method.

\section{1. Введение}

В настоящее время нефтяная промышленность характеризуется увеличением запасов трудноизвлекаемой нефти, доля которых растет и в России. Одной из разновидностей трудноизвлекаемых запасов являются месторождения высоковязких нефтей. Установлено, что в таких коллекторах нефть проявляет вязкопластические свойства, закон фильтрации отклоняется от линейного закона Дарси [1]. Широкое применение при разработке месторождений тяжелых нефтей нашли тепловые методы увеличения нефтеотдачи (МУН).

Численное моделирование вариантов разработки месторождений высоковязких нефтей с использованием тепловых методов увеличения нефтеотдачи (МУН) имеет ряд трудностей, связанных с необходимостью учета вязкопластичной реологии нефти, расчетом фазового равновесия смеси, высоким контрастом подвижности газовой фазы и жидких фаз. Использование традиционных конечно-объемных методов приводит к задачам с большой 
вычислительной сложностью. Поэтому представляет интерес использование методов расщепления при моделировании задач композиционной нелинейной фильтрации. Одними из наиболее распространенных методов являются методы линий тока.

Основная идея разработанных методов линий тока [2-7] заключается в решении уравнения для давления на исходной сетке с последующим интегрированием уравнений для фазовых насыщенностей и температуры вдоль линий тока. Схема расщепления системы уравнений фильтрации (в неизотермическом случае) по физическим процессам приведена на рис.1.

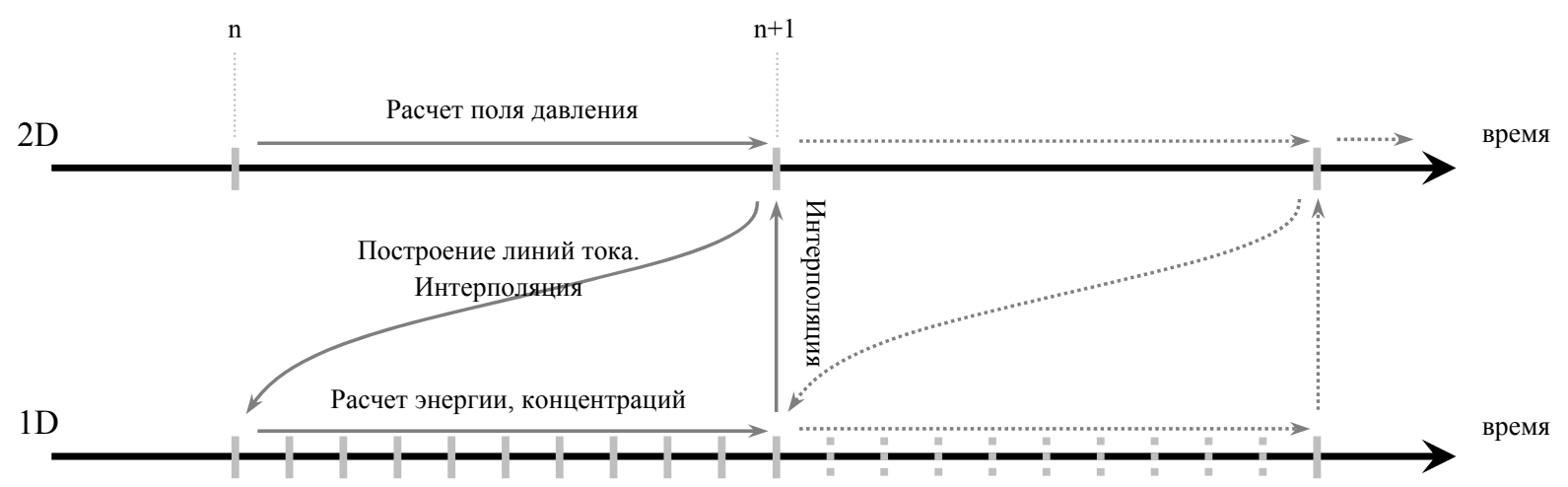

Рис.1. Схема моделирования методом линий тока.

Во всех упомянутых работах для описания фильтрации использовался линейный закон Дарси. Фильтрация высоковязких нефтей описывается нелинейными законами, параметры которых зависят от температуры [8], в результате чего для корректного определения распределения давления в пласте при решении системы уравнений фильтрации необходимо учитывать изменение температуры. Поэтому стандартный подход с использованием линий тока может давать неверное распределение давления и, как следствие, неправильную геометрию линий тока.

Особенностью численного метода, предлагаемого в работе, является использование линий тока для решения системы уравнений многофазной многокомпонентной фильтрации на следующих временных шагах, а не для определения температуры и насыщенности на текущем временном слое при фиксированном давлении. В результате предложенный подход позволяет учесть зависимость реологии нефти от температуры.

\section{2. Математическая модель}

2.1. Уравнения баланса. Основу математической композиционной модели неизотермической фильтрации флюида, состоящего из $N_{c}$ компонентов при наличии $N_{p}$ фаз, составляют законы сохранения массы компонентов 


$$
\frac{\partial N_{i}}{\partial t}-\nabla \cdot \sum_{j=1}^{N_{p}} \alpha_{j} \frac{k_{\mathrm{res}} k_{r j} \rho_{j}}{\mu_{j}} c_{i j} \nabla p=q_{i}
$$

а также закон сохранения энергии:

$$
\frac{\partial e}{\partial t}-\nabla \cdot\left(\sum_{i=1}^{N_{c}} \sum_{j=1}^{N_{p}} \alpha_{j} \frac{k_{r e s} k_{r j} \rho_{j}}{\mu_{j}} c_{i j} h_{i j} \nabla p+\bar{\lambda} \nabla T\right)=q_{e} .
$$

Здесь $q_{i}$ - мольный расход $i$-го компонента на единицу объема, $k_{\text {res }}-$ абсолютная проницаемость пласта, $k_{r j}, \rho_{j}, \mu_{j}$ - соответственно относительная фазовая проницаемость (ОФП), плотность и вязкость фазы $j$ (водная, нефтяная или газовая фаза), $p$ - давление, $e$ - плотность внутренней энергии, $q_{e}-$ объемная плотность источников тепла (энергии), $h_{i j}$ - молярная энтальпия компонента $i$ в фазе $j, c_{i j}$ - мольная доля компонента $i$ в фазе $j, T$ - температура, $\bar{\lambda}$ - осредненный коэффициент теплопроводности смеси. Молярная концентрация $N_{i}$ компонента $i$ вычисляется следующим образом:

$$
N_{i}=\varphi \sum_{j=1}^{N_{p}} c_{i j} S_{j} \rho_{j}
$$

где $\varphi$ - пористость, $S_{j}$ - объемная доля фазы $j$ в поровом пространстве.

В работе капиллярное давление между фазами полагалось равным нулю, при написании уравнений (1) и (2) использовалась связь скорости фильтрации $v_{j}$ и градиента давления:

$$
v_{j}=-\alpha_{j} \frac{k_{\mathrm{res}} k_{r j}}{\mu_{j}} \nabla p .
$$

Для моделирования нелинейных законов фильтрации в (4) введен вспомогательный коэффициент $\alpha_{j}$, который для нефтяной фазы определяется как

$$
\alpha_{\mathrm{o}}= \begin{cases}1-\frac{\gamma}{|\nabla p|}, & |\nabla p| \geq \gamma \\ 0, & |\nabla p|<\gamma\end{cases}
$$

где $\gamma$ - значение предельного градиента давления. Представленный закон фильтрации является наиболее распространенным при описании течения высоковязких нефтей [9] и поэтому используется в данной работе. Фильт- 
рация воды и газа подчиняется закону Дарси, поэтому $\alpha_{w}=\alpha_{g}=1$. Как было отмечено ранее, реологические характеристики нефти сильно зависят от температуры. Поэтому в рассматриваемую математическую модель включена зависимость предельного градиента от температуры [8].

Уравнение для давления имеет вид

$$
\begin{gathered}
\frac{\partial S_{\Sigma}}{\partial p} \frac{\partial p}{\partial t}+\frac{\partial S_{\Sigma}}{\partial e} \nabla \cdot\left(\sum_{i=1}^{N_{c}} \sum_{j=1}^{N_{p}} \alpha_{j} \frac{k_{\mathrm{res}} k_{r j} \rho_{j}}{\mu_{j}} c_{i j} h_{i j} \nabla p+\bar{\lambda} \nabla T\right)+ \\
+\sum_{i=1}^{N_{c}}\left(\frac{\partial S_{\Sigma}}{\partial N_{i}} \nabla \cdot \sum_{j=1}^{N_{p}} \alpha_{j} \frac{k_{\mathrm{res}} k_{r j} \rho_{j}}{\mu_{j}} c_{i j} \nabla p\right)+\frac{\partial S_{\Sigma}}{\partial e} q_{e}+\sum_{i=1}^{N_{c}}\left(\frac{\partial S_{\Sigma}}{\partial N_{i}} q_{i}\right)=0 .
\end{gathered}
$$

При его выводе используется условие совместности объемов фаз $S_{\Sigma}=1$. Частные производные $\partial S_{\Sigma} / \partial N_{i}, \partial S_{\Sigma} / \partial p, \partial S_{\Sigma} / \partial e$ характеризуют зависимость объема порового пространства, занятого флюидами от мольных концентраций компонентов, давления и энергии, соответственно.

2.2. Замыкающие соотношения. Помимо коэффициентов $c_{i j}$ в систему уравнений фильтрации (1), (2) входят свойства фаз, такие как плотность, вязкость, молярная энтальпия, коэффициент теплопроводности. Они определяются не только компонентным составом, но и свойствами каждого компонента. Для расчета свойств компонентов и фаз использованы распространенные в программном обеспечении соотношения [10,11].

2.3. Начальные и граничные условия. Для задания начальных условий используются распределения давления, температуры и мольных долей компонентов. Из этих данных после расчета фазового равновесия можно получить распределение начальных мольных концентраций, фазовых насыщенностей и плотностей внутренних энергий.

При моделировании пласт предполагается замкнутым, поэтому при решении исходной двумерной задачи на границах расчетной области заданы условия непротекания всех фаз, а также нулевой поток энергии. В случае моделирования утечек тепла через верхнюю и нижнюю границы пласта условие нулевого потока энергии на границе не используется. Вместо него используется условие постоянной температуры на боковой грани расчетной области.

\section{3. Численный метод}

Рассматривается плоская двумерная задача фильтрации в прямоугольном пласте размером $X_{0} \times Y_{0}$, толщина которого $\Delta \mathrm{z}$ постоянна. Введена 
равномерная двумерная сетка с $N_{X} \times N_{Y}$ ячейками, размер которых равен $\Delta x=X_{0} / N_{X}, \Delta y=Y_{0} / N_{Y}$. Для удобства аппроксимации граничных условий снаружи расчетной области добавлен ряд фиктивных ячеек. Координаты границ ячеек вдоль оси ОX $x_{l+1 / 2}, l=1, \ldots, N_{X}+1$ определяются в процессе генерации сетки, координаты же центров ячеек определяются из соотношения $x_{l}=\left(x_{l-1 / 2}+x_{l+1 / 2}\right) / 2$, где $l=2, \ldots, N_{X}+1$ (аналогичным образом для направления OY).

Определение траектории линии тока происходит последовательно для каждой ячейки двумерной (глобальной) сетки. Полученная в результате сетка на линиях тока является неравномерной, так как узлы ячеек на линиях тока соответствуют точкам пересечения линий тока с границами ячеек двумерной сетки. Пример расположения ячеек глобальной сетки и узлов сетки на линии тока приведен на рис.2.

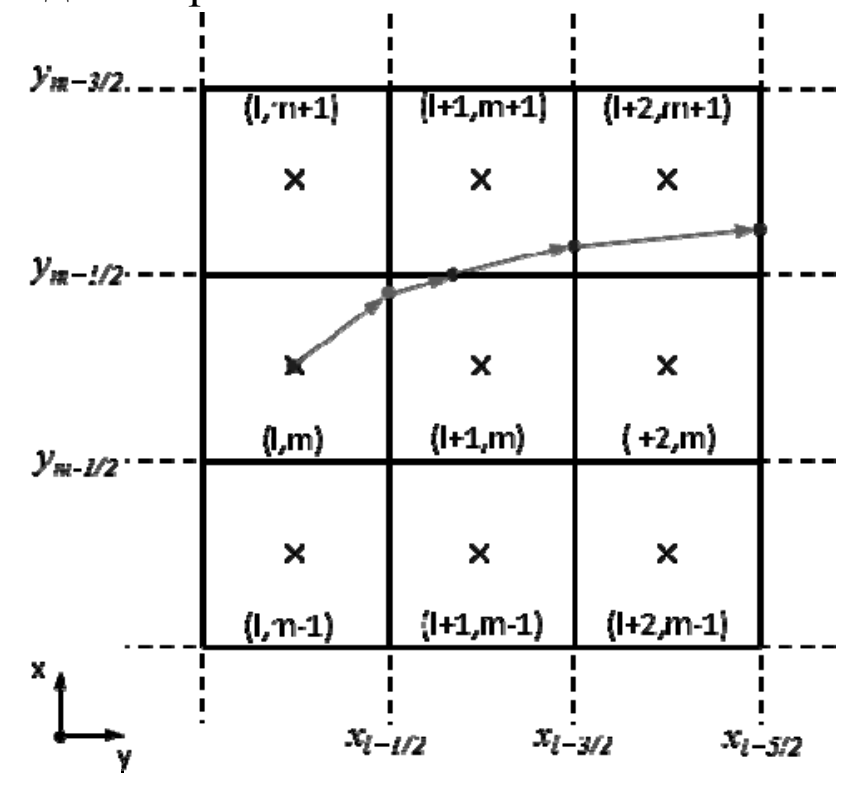

Рис.2. Расположение узлов двумерной сетки и узлов сетки на линии тока.

В вектор основных переменных входит давление, плотность внутренней энергии и молярные концентрации компонентов, т.е. $\mathbf{U}=\left(p, e, N_{i}\right)^{T}$. Общая схема алгоритма выглядит следующим образом:

1. Решение системы уравнений (1), (2) на двумерной сетке в течение времени установления. Известно значение вектора основных переменных на $n$-м слое, т.е. $\mathbf{U}^{n}=\left(p^{n}, e^{n}, N_{i}^{n}\right)^{T}$.

1.1. Учет вклада теплопроводности в плотность внутренней энергии $\left(p^{n}, e^{n}, N_{i}^{n}\right)^{T} \rightarrow\left(p^{n}, \hat{e}, N_{i}^{n}\right)^{T}$.

1.2. Решение уравнения для давления (2.7) $\left(p^{n}, \hat{e}, N_{i}^{n}\right)^{T} \rightarrow\left(p^{*}, \hat{e}, N_{i}^{n}\right)^{T}$. 
1.3. Расчет плотности внутренней энергии и молярных концентраций $\left(p^{*}, \hat{e}, N_{i}^{n}\right)^{T} \rightarrow\left(p^{*}, e^{*}, N_{i}^{*}\right)^{T}$.

1.4. Расчет равновесной температуры, компонентного и фазового состава смеси $\left(p^{*}, e^{*}, N_{i}^{*}\right)^{T} \rightarrow\left(T^{*}, S_{j}^{*}, c_{i j}^{*}\right)^{T}$.

1.5. Проверка условия совместности объемов фаз $\left|\sum_{j} S_{j}^{*}-1\right|<\varepsilon$, где $\varepsilon-$ заданная точность.

1.6. При выполнении условия совместности $\left(p^{n+1}, e^{n+1}, N_{i}^{n+1}\right)^{T}=$ $=\left(p^{*}, e^{*}, N_{i}^{*}\right)^{T}$, после чего проводится расчет поля скоростей фильтрации $u_{t}$. В противном случае $\tau=\tau / 2$ и переходим к п. 1.1.

2.

Применение линий тока.

2.1. Построение линий тока $\left(u_{t}^{n+1}, x_{l \pm 1 / 2}, y_{m \pm 1 / 2}\right)^{T} \rightarrow x_{\beta, i}$, где $\beta$ - номер линии тока.

2.2. Учет вклада теплопроводности в плотность внутренней энергии $\left(p^{n+1}, e^{n+1}, N_{i}^{n+1}\right)^{T} \rightarrow\left(p^{n+1}, \hat{e}, N_{i}^{n+1}\right)^{T}$.

2.3. Интерполяция основных переменных на линии тока $\left(p^{n+1}, \hat{e}, N_{i}^{n+1}\right)^{T} \rightarrow\left(p_{\beta}^{n+1}, \hat{e}_{\beta}, N_{\beta, i}^{n+1}\right)^{T}$.

2.4. Решение системы уравнений (1), (2) на линиях тока без учета теплопроводности $\left(p^{n+1}, \hat{e}, N_{i}^{n+1}\right)^{T} \rightarrow\left(p_{\beta}^{n+2}, e_{\beta}^{n+2}, N_{\beta, i}^{n+2}\right)^{T}$.

2.5. В случае существенного отличия давления между соседними линиями тока проводится интерполяция решения с линий тока на двумерную сетку $\left(p_{\beta}^{n+2}, e_{\beta}^{n+2}, N_{\beta, i}^{n+2}\right)^{T} \rightarrow\left(p^{n+2}, e^{n+2}, N_{i}^{n+2}\right)^{T}$, переход к п.1. Если давление отличается слабо, на двумерную сетку интерполируется только плотность внутренней энергии, переход к п. 2.2.

Подробнее рассмотрим основные пункты, приведенные в схеме алгоритма.

\section{1. Решение уравнений фильтрации на двумерной сетке.}

3.1.1. Определение вектора основных переменных на следующем временном слое. Численное решение уравнений фильтрации (1), (2) осуществляется методом IMРЕС (неявный по давлению, явный по концентрациям компонентов), идея которого заключается в том, что при аппроксимации уравнения (6) давление учитывается на $n+1$ временном слое, а остальные переменные учитываются явно. 
В уравнение для давления (6) входит слагаемое, ответственное за кондуктивный поток энергии. Так как в вектор основных переменных вместо температуры входит плотность внутренней энергии, целесообразно использовать явное значение температуры при численном решении этого уравнения, что, однако, ухудшает устойчивость метода. Для сохранения устойчивости применен метод расщепления по физическим процессам: перед решением уравнения для давления проводится корректировка внутренней энергии за счет теплопроводности, после чего решается уравнение для давления с обновленным значением внутренней энергии, но без слагаемого в самом уравнении. Таким образом, уравнение для давления имеет вид

$$
\begin{gathered}
\frac{\partial S_{\Sigma}}{\partial p} \frac{\partial p}{\partial t}+\frac{\partial S_{\Sigma}}{\partial e} \nabla \cdot\left(\sum_{i=1}^{N_{c}} \sum_{j=1}^{N_{p}} \alpha_{j} \frac{k_{\mathrm{res}} k_{r, j} \rho_{j}}{\mu_{j}} c_{i j} h_{i, j} \nabla p\right)+ \\
+\sum_{i=1}^{N_{c}}\left(\frac{\partial S_{\Sigma}}{\partial N_{i}} \nabla \cdot \sum_{j=1}^{N_{p}} \alpha_{j} \frac{k_{\mathrm{res}} k_{r, j} \rho_{j}}{\mu_{j}} c_{i j} \nabla p\right)+\frac{\partial S_{\Sigma}}{\partial e} q_{e}+\sum_{i=1}^{N_{c}}\left(\frac{\partial S_{\Sigma}}{\partial N_{i}} q_{i}\right)=0 .
\end{gathered}
$$

Расчет пространственных производных от суммарной насыщенности $S_{\Sigma}$ осуществляется по формуле с центральной разностью. Дискретизация уравнения для давления на неявном пятиточечном конечно-разностном шаблоне (в терминах невязки) на равномерной сетке (индексы ячеек $l$ и $m$ ) имеет вид

$$
\begin{aligned}
R_{l, m} & =c_{l, m} p_{l+1, m}^{n+1}+f_{l, m} p_{l, m+1}^{n+1}+a_{l, m} p_{l, m}^{n+1}+b_{l, m} p_{l-1, m}^{n+1}+ \\
& +g_{l, m} p_{l, m-1}^{n+1}-d_{l, m}^{n}=0 .
\end{aligned}
$$

Входящие в уравнение (5) коэффициенты равны

$$
\begin{gathered}
c_{l, m}=\left(\frac{\partial S_{\Sigma}}{\partial e}\right)_{l, m}^{n} T E X_{l+1 / 2, m}+\sum_{i=1}^{N_{c}}\left[\left(\frac{\partial S_{\Sigma}}{\partial N_{i}}\right)_{l, m}^{n} T X_{i, l+1 / 2, m}\right], \\
b_{l, m}=\left(\frac{\partial S_{\Sigma}}{\partial e}\right)_{l, m}^{n} T E X_{l-1 / 2, m}+\sum_{i=1}^{N_{c}}\left[\left(\frac{\partial S_{\Sigma}}{\partial N_{i}}\right)_{l, m}^{n} T X_{i, l-1 / 2, m}\right], \\
f_{l, m}=\left(\frac{\partial S_{\Sigma}}{\partial e}\right)_{l, m}^{n} T E Y_{l, m+1 / 2}+\sum_{i=1}^{N_{c}}\left[\left(\frac{\partial S_{\Sigma}}{\partial N_{i}}\right)_{l, m}^{n} T Y_{i, l, m+1 / 2}\right], \\
g_{l, m}=\left(\frac{\partial S_{\Sigma}}{\partial e}\right)_{l, m}^{n} T E Y_{l, m-1 / 2}+\sum_{i=1}^{N_{c}}\left[\left(\frac{\partial S_{\Sigma}}{\partial N_{i}}\right)_{l, m}^{n} T Y_{i, l, m-1 / 2}\right],
\end{gathered}
$$




$$
\begin{aligned}
& \varphi_{l, m}=\frac{V_{l, m}}{\tau}\left(\frac{\partial S_{\Sigma}}{\partial p}\right)_{l, m}^{n}, \\
& a_{l, m}=-\left(c_{l, m}+b_{l, m}+f_{l, m}+g_{l, m}\right)+\varphi_{l, m}, \\
& d_{l, m}=\varphi_{l, m} p_{l, m}^{n}-\left(\frac{\partial S_{\Sigma}}{\partial p}\right)_{l, m}^{n} Q_{e, l, m}^{n+1}-\sum_{i=1}^{N_{c}}\left[\left(\frac{\partial S_{\Sigma}}{\partial N_{i}}\right)_{l, m}^{n} Q_{i, l, m}^{n+1}\right],
\end{aligned}
$$

где $\tau$ - шаг по времени и введены коэффициенты межблочной проводимости для потока энергии:

$$
T E X_{l \pm 1 / 2, m}=\left(\sum_{i=1}^{N_{c}} \sum_{j=1}^{N_{p}} \alpha_{j} \frac{k_{\mathrm{res}} k_{r j} \rho_{j}}{\mu_{j}} c_{i j} h_{j}\right)_{l \pm 1 / 2, m} \frac{\Delta y_{m} \Delta z}{\Delta x_{l \pm 1 / 2}}
$$

и для потока массы:

$$
T X_{i, l \pm 1 / 2, m}=\left(\sum_{j=1}^{N_{p}} \alpha_{j} \frac{k_{\mathrm{res}} k_{r j} \rho_{j}}{\mu_{j}} c_{i j}\right)_{l \pm 1 / 2, m} \frac{\Delta y_{m} \Delta z}{\Delta x_{l \pm 1 / 2}} .
$$

Аналогичным образом записываются коэффициенты межблочной проводимости для направления вдоль оси ОY. Для расчета межблочных проводимостей применялась аппроксимация вверх по потоку.

Полученная система уравнений (8) имеет пятидиагональную матрицу. Для ее численного решения применен полинейный метод Гаусса-Зейделя [12].

Ввиду того, что коэффициенты $c_{l, m}, b_{l, m}, g_{l, m}, f_{l, m}, a_{l, m}$ зависят от давления, для их корректного учета был применен метод Ньютона с численным вычислением элементов матрицы Якоби (метод переменных секущих).

При написании аппроксимационных соотношений уравнения для давления не были указаны временные слои для источниковых слагаемых. Для учета скважин применен линеаризованный метод:

$$
Q_{j, l, m}^{n+1} \approx Q_{j, l, m}^{n}+\left(\frac{\partial Q_{j, l, m}}{\partial p_{l, m}}\right)^{n}\left(p_{l, m}^{n+1}-p_{l, m}^{n}\right) .
$$

Объемный расход $Q_{j}$ фазы $j$ рассчитывается с помощью модели Писмана.

После подстановки выражения для дебита скважины в дискретизацию уравнения для давления в коэффициент $a_{l, m}$ добавится слагаемое 
$\left(\partial Q_{j, l, m} / \partial p_{l, m}\right)^{n}$, остальные слагаемые в формуле (18) участвуют в расчете коэффициента $d_{l, m}$.

После определения давления явно рассчитывается внутренняя энергия и молярные концентрации компонентов:

$$
\begin{aligned}
& e_{l, m}^{n+1}=e_{l, m}^{n}+\tau\left[\begin{array}{l}
\operatorname{TEX} X_{l+1 / 2, m}\left(p_{l+1, m}^{n+1}-p_{l, m}^{n+1}\right)-T E X_{l-1 / 2, m}\left(p_{l, m}^{n+1}-p_{l-1, m}^{n+1}\right)+ \\
+T E Y_{l, m+1 / 2}\left(p_{l, m+1}^{n+1}-p_{l, m}^{n+1}\right)-T E Y_{l, m-1 / 2}\left(p_{l, m}^{n+1}-p_{l, m-1}^{n+1}\right)
\end{array}\right], \\
& N_{i, l, m}^{n+1}=N_{i, l, m}^{n}+\tau\left[\begin{array}{c}
T X_{i, l+1 / 2, m}\left(p_{l+1, m}^{n+1}-p_{l, m}^{n+1}\right)-T X_{i, l-1 / 2, m}\left(p_{l, m}^{n+1}-p_{l-1, m}^{n+1}\right)+ \\
T Y_{i, l, m+1 / 2}\left(p_{l, m+1}^{n+1}-p_{l, m}^{n+1}\right)-T Y_{i, l, m-1 / 2}\left(p_{l, m}^{n+1}-p_{l, m-1}^{n+1}\right)
\end{array}\right] .
\end{aligned}
$$

Приведенный алгоритм вычисления накладывает ограничение на величину временного шага, поэтому для его контроля анализировалось относительное изменение внутренней энергии и молярных концентраций:

$$
\max _{l, m}\left|\left(e_{l, m}^{n+1}-e_{l, m}^{n}\right) / e_{l, m}^{n}\right|<0.5, \max _{i, l, m}\left|\left(N_{i, l, m}^{n+1}-N_{i, l, m}^{n}\right) / N_{i, l, m}^{n}\right|<0.1 .
$$

Здесь константы определены по результатам тестовых расчетов.

3.1.2. Расчет фазового равновесия. Одними из величин, входящих в систему уравнений фильтрации (1), (2), являются коэффициенты $c_{i j}$, которые характеризуют мольную долю компонента $i$ в фазе $j$. Для их определения по заданным значениям давления, температуры и молярным концентрациям компонентов выполняется расчет фазового равновесия.

Пусть $L_{j}$ - мольная доля фазы $j$ в поровом пространстве, $n_{i}$ - молярные доли компонентов (1 - водный компонент, 2 - легкая нефть, 3 - тяжелая нефть). Тогда материальный баланс компонентов определяется следующей системой уравнений:

$$
\sum_{j=1}^{N_{p}} L_{j} c_{i j}=n_{i} .
$$

Условия фазового равновесия описаны в терминах констант фазового равновесия (коэффициентов распределения)

$$
K_{1}=c_{1, \mathrm{~g}} / c_{1, \mathrm{w}}, \quad K_{2}=c_{2, \mathrm{~g}} / c_{2, \mathrm{o}}, \quad K_{3}=c_{3, \mathrm{~g}} / c_{3, \mathrm{o}} .
$$

Из распределения компонентов по фазам и условий совместности мольных долей фаз видно, что 


$$
c_{1, \mathrm{w}}=1, \quad \sum_{i=2,3} c_{i, \mathrm{o}}=1, \quad \sum_{i=1}^{N_{c}} c_{i, \mathrm{~g}}=1, \quad \sum_{j=1}^{N_{p}} L_{j}=1 .
$$

В работе принято, что константы фазового равновесия являются функциями давления и температуры и не зависят от фазового состава. Для вычисления констант фазового равновесия использована корреляция Вильсона:

$$
K_{i}=\frac{p_{\mathrm{c}, i}}{p} \exp \left(5.372697\left(1+\omega_{i}\right)\left(1-\frac{T_{\mathrm{c}, i}}{T}\right)\right),
$$

где $p_{\mathrm{c}}, T_{\mathrm{c}}$ - критические давление и температура компонента, $\omega$ - ацентрический фактор [13].

В расчете фазового равновесия неизвестными величинами являются мольные доли компонентов в фазах $c_{i j}$, а также мольные доли фаз $L_{j}(9$ неизвестных). Система (21)-(23) состоит из 10 уравнений. Учитывая, что $\sum_{i=1}^{N_{c}} n_{i}=1$, одно из уравнений (23) можно исключить из системы. Как результат, представленная математическая модель допускает корректный расчет фазового равновесия.

В зависимости от того, в каких фазах присутствуют рассматриваемые компоненты, возможно несколько состояний при фазовом равновесии, а именно: присутствие всех компонентов во всех фазах, присутствие всех компонентов только в жидкой фазе, только в газовой фазе, полное испарение водной фазы, полное испарение нефтяной фазы. Во всех случаях полученная система уравнений может быть решена аналитически или численно. Критерии существования каждой равновесного состояния получаются из следующих условий:

$$
L_{j}, c_{i j} \in[0,1],
$$

в которые подставляются соотношения для компонентного и фазового состава, полученные для каждой ветки фазового равновесия.

В случае отсутствия одного из компонентов изменение мольной доли фазы происходит скачкообразно, как следствие, итерации метода Ньютона не сойдутся. Поэтому в работе при дискретизации уравнений коэффициент межблочной проводимости для потока массы компонента корректируется следующим образом: 


$$
T X_{i, l \pm 1 / 2, m}= \begin{cases}T X_{i, l \pm 1 / 2, m}\left(1-10^{-4} / n_{i}{ }^{2}\right), & n_{i} \geq 10^{-4} \\ 0, & n_{i}<10^{-4},\end{cases}
$$

в результате чего мольная доля компонента в ячейке не будет становиться меньше $10^{-4}$. Такой подход позволяет сохранить сходимость метода Ньютона, причем возмущение в численном решении будет незначительным.

3.1.3. Учет теплопроводности. При решении системы (1), (2) теплопроводность учитывается на отдельном этапе. Перед решением уравнения для давления проводится корректировка внутренней энергии по уравнению

$$
\frac{\partial e}{\partial T} \frac{\tilde{T}-T^{n}}{\tau}=\operatorname{div} \kappa \nabla \tilde{T}+q_{h},
$$

где $\kappa$ - коэффициент температуропроводности, $q_{h}$ - источниковое слагаемое, которое складывается из утечек тепла через границы пласта и мощности (в единице объема) нагревательного элемента, спущенного в скважину. Численное решение уравнения (27) осуществляется неявным методом на пятиточечном разностном шаблоне, аналогичном тому, который использовался для решения уравнения для давления.

Для учета теплопотерь через кровлю и подошву пласта была применена полуаналитическая методика, описанная в [14].

После определения вспомогательной температуры $\tilde{T}$ пересчитывается внутренняя энергия:

$$
\tilde{e}_{l, m}^{n}=e_{l, m}^{n}+\tau \cdot \operatorname{div} \kappa \nabla \tilde{T} .
$$

Полученное значение энергии $\tilde{e}_{l, m}^{n}$ используется при решении системы методом IMPEC.

3.2. Метод линий тока. В работе предложен метод, заключающийся в использовании линий тока для решения уравнений фильтрации на следующих временных шагах, а не для уточнения решения на текущем временном слое. При этом сетка на линиях тока должна перестраиваться в случае существенного отличия решения на соседних линиях тока. Для этого после нарушения условия сходимости решения между линиями тока, выполняется интегрирование уравнений на исходной декартовой сетке в течение времени установления, после чего выполняется перестроение линий тока и т.д. Таким образом, интегрирование системы уравнений фильтрации рассматривается как совокупность квазиравновесных состояний, между которыми 
уравнение решается на сетке из линий тока. В качестве времени установления используется время пьезопроводности:

$$
\Delta t_{\text {global }}=L^{2} / \max _{j}\left(k_{\text {res }} k_{r j} / \phi \mu_{j} c_{f, j}\right),
$$

где $L$ - характерный размер расчетной области, $c_{f, j}$ - сжимаемость фазы $j$, максимум рассчитывается по всем фазам.

3.2.1. Построение линий тока. Для построения линий тока применена процедура, предложенная Поллоком [15]. Основное предположение - линейное распределение компонентов скорости фильтрации.

Выбор метода Поллока для построения линий тока обусловлен простотой реализации ввиду наличия аналитического выражения для координат частиц на линии тока. Кроме того, в процессе построения рассчитывается так называемое время пролета (time of flight - TOF) - время, необходимое частице для преодоления расстояния между двумя сегментами линии тока. По определению:

$$
\tau_{\mathrm{TOF}}(s)=\int_{0}^{s} \varphi(\xi) /\left|\vec{u}_{t}\right| d \xi
$$

где $s$ - координата вдоль линии тока. В предположении постоянства потока вдоль трубки тока, ассоциированной с линией тока, можно получить выражение для объема трубки тока $V_{\beta}$ :

$$
V_{\beta}=\int_{\mathrm{s}_{\text {in }}}^{s_{\text {out }}} \varphi_{\beta}(\xi) A_{\beta}(\xi) d \xi=\int_{\mathrm{s}_{\text {in }}}^{s_{\text {out }}} q_{\beta}(\xi) \tau_{T O F, \beta}(\xi) d \xi,
$$

где $A_{\beta}(\xi)$ - площадь поперечного сечения трубки тока на участке от $s_{i n}$ до $s_{\text {out }}, q_{\beta}(\xi)$ - расход вдоль трубки тока. Таким образом, рассчитав распределение времени пролета частицы вдоль линии тока и зная расход вдоль ассоциированной трубки тока, можно вычислить геометрические размеры сетки на трубках тока. Основное преимущество такого подхода - отсутствие необходимости отслеживать положение границ трубки тока, что крайне затруднительно в трехмерном случае. Для сегмента с индексом $i$ ширина ассоциированной трубки тока вычисляется как:

$$
\Delta y_{\beta, i}=\frac{q_{\beta} \tau_{\mathrm{TOF}, \beta, i}}{\Delta z \Delta x_{\beta, i}} .
$$


При генерации трубок тока предполагается, что вблизи скважины они распределены равномерно, причем расход для каждой линии тока, пересекающей одну и ту же грань ячейки со скважиной, одинаков. Вычисление расхода основывается на доли, которую составляет поток через рассматриваемую грань относительно суммарного потока из ячейки со скважиной. Расход через рассматриваемую трубку тока равен произведению этой доли на суммарный дебит (закачку) фаз скважины.

3.2.2. Интерполяция решения на сетку из линий тока. Перед решением системы уравнений фильтрации на сетке из линий тока необходимо определить начальное распределение неизвестных на соответствующей сетке. Для этого применена биквадратичная интерполяция, схема выбора узлов для которой приведена на рис.2. Здесь точками отмечена сетка на линиях тока, серым - узел между ячейками $(l, m)$ и $(l+1, m)$, в котором необходимо определить значение основных переменных, узлы интерполяции отмечены крестиком на декартовой сетке.

Стоит отметить, что предположение линейного распределения скорости фильтрации внутри декартовой ячейки нарушается вблизи скважины, в результате чего линии тока отходят от границы ячейки, содержащей скважину [2-7]. Такой подход вносит дополнительную погрешность аппроксимации граничных условий, особенно если принимать во внимание размер расчетных ячеек (порядка сотни метров) при полномасштабном моделировании. Для ячеек одномерной сетки, примыкающих к скважине, ширина $\Delta y_{3 / 2}$ рассчитывается с использованием межблочной проводимости:

$$
\begin{aligned}
& q_{\beta}=T X_{3 / 2}\left(p_{2}-p_{1}\right)=\lambda X_{3 / 2} \cdot \frac{\Delta z \Delta y_{3 / 2}}{x_{2}-x_{1}}\left(p_{2}-p_{1}\right)=\frac{\omega_{l+1 / 2, m} Q_{l, m}}{n_{l+1 / 2, m}}, \\
& \Delta y_{3 / 2}=q_{\beta} \cdot \frac{x_{2}-x_{1}}{\Delta z \lambda X_{3 / 2}\left(p_{2}-p_{1}\right)},
\end{aligned}
$$

где $n_{l+1 / 2, m}$ - количество линий тока, выходящих из ячейки $(l, m)$ и пересекающих одну и ту же грань (в данном случае грань с координатами $x_{l+1 / 2}$, $\left.y_{m}\right), Q_{l, m}$ - дебит всех фаз в скважине, $\omega_{l+1 / 2, m}$ - доля, которую составляет поток через грань $x_{l+1 / 2}, y_{m}$ относительно суммарного потока из ячейки $x_{l}$, $y_{m}, \lambda X_{3 / 2}$ - суммарная подвижность фаз на границе расчетной ячейки на трубке тока. Давление $p_{1}$ соответствует давлению в скважине, давление $p_{2}$ определяется в результате интерполяции с глобальной сетки на трубку тока. 
Полученная в результате сетка на линиях тока является неравномерной, так как узлы соответствуют точкам пересечения с глобальной сеткой. Численное решение уравнений фильтрации на такой сетке налагает ограничения на допустимую величину временного шага. Для улучшения сходимости решения исходная сетка на линиях тока заменяется на равномерную, для которой пересчитывается время пролета между соседними узлами, а также геометрические характеристики новой сетки. После чего значения основных переменных интерполируются на равномерную сетку также по квадратичному алгоритму.

\subsection{3. Учет теплопроводности и решение уравнений фильтрации на} линиях тока. Численное решение уравнений фильтрации на линиях тока осуществляется методом IMРЕС, аналогичным тому, который использовался для решения системы уравнений на двумерной сетке.

Моделирование теплопроводности при решении системы уравнений вдоль линий тока производится на отдельном этапе, перед интерполяцией решения с двумерной сетки на трубки тока. Пересчет внутренней энергии и температуры происходит так же, как и в двумерном случае, путем решения вспомогательных уравнений (27), (28) на интервале времени, в течение которого фильтрация рассчитывается на линиях тока. Полученное значение внутренней энергии используется при решении системы на трубках тока.

3.2.4. Интерполяция решения на глобальную сетку. После решения уравнений фильтрации на трубках тока полученные значения интерполируются на исходную неравномерную сетку на трубках тока. Затем исследуется сходимость решения: если давление между соседними линиями тока, пересекающими одинаковую грань ячейки, отличается менее чем на заданную величину $\varepsilon$ (в расчетах используется значение $10 \%$ ), то решение на сетке из линий тока продолжается. В противном случае решение интерполируется на глобальную сетку. Для этого используется алгоритм, описанный в [16]. Значение в узле двумерной сетки вычисляется как средневзвешенное значение по трубкам тока, проходящим через рассматриваемую ячейку, где в качестве веса используется объем сегмента трубки тока:

$$
\mathbf{U}_{l, m}=\sum_{\beta=1}^{n_{s}} q_{\beta} \tau_{\mathrm{TOF}, \beta} \vec{U}_{\beta} / \sum_{\beta=1}^{n_{s}} q_{\beta} \tau_{\mathrm{TOF}, \beta},
$$

где $\mathbf{U}_{l, m}$ - вектор неизвестных в ячейке $(l, m), n_{s}$ - количество линий тока, пересекающих рассматриваемую ячейку.

Ввиду того что вблизи скважины распределение скорости фильтрации является нелинейным, определение значения давления в ячейке двумерной 
сетки, содержащей скважину, производится отдельным методом, без использования времени пролета. Зная значение расходов для каждой трубки тока, можно определить дебит скважины, после чего по формуле Писмана можно определить давление в ячейке двумерной сетки.

\section{4. Результаты моделирования}

Для проверки реализации метода линий тока были проведены тестовые расчеты на элементе симметрии пятиточечной системы разработки, размером $150 \times 150$ м (рис.3). В левом верхнем углу расчетной области располагается нагнетательная скважина, закачивающая горячую воду заданной температуры. В правом нижнем углу задана добывающая скважина. Контроль скважин осуществляется по постоянному забойному давлению. В начальный момент времени пласт насыщен водой и нефтью. Расчет проводился на двумерной сетке, для которой $\Delta x=\Delta y=6 \mathrm{M}$, размер расчетной ячейки на линиях тока составлял 10 м. При моделировании использовалось 37 линий тока. Результаты расчетов сопоставлены с результатами моделирования с использованием пятиточечного и девятиточечного разностных шаблонов (рис.4).

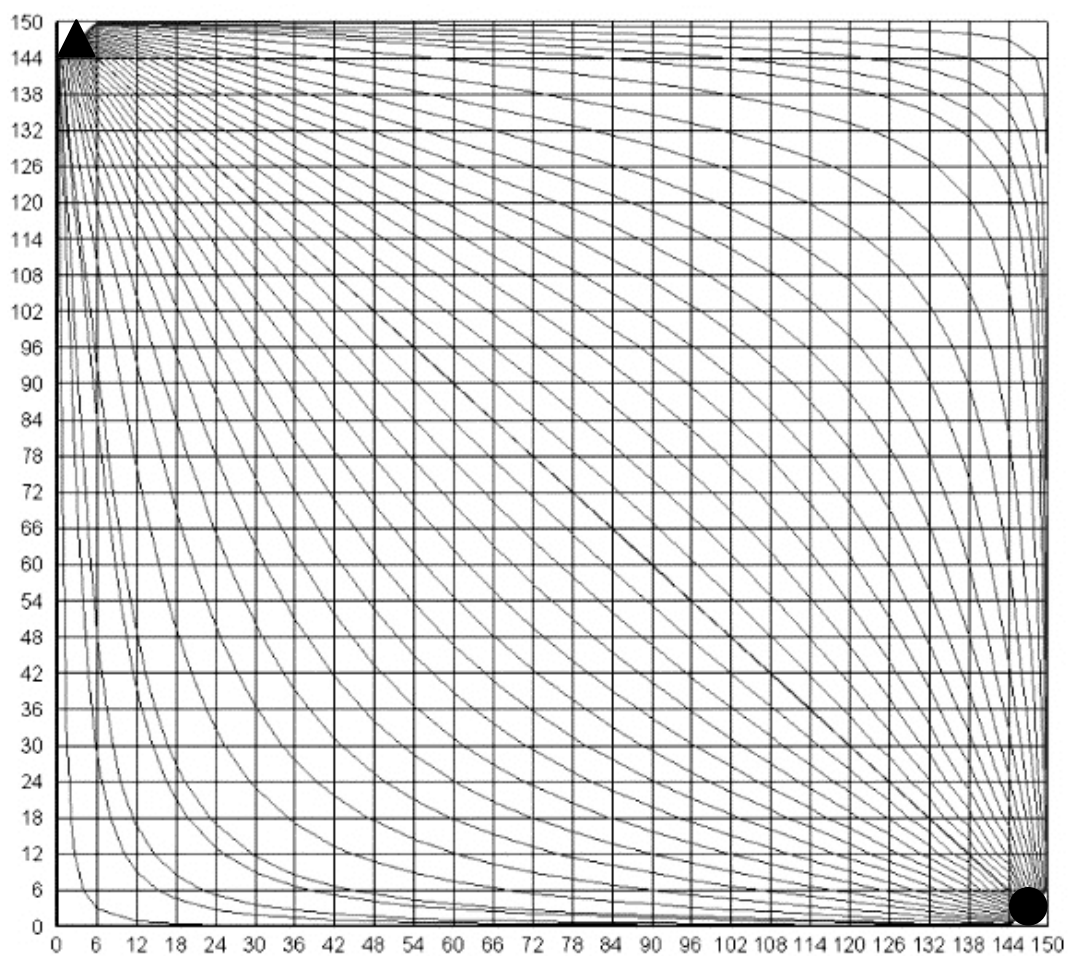

Рис.3. Расчетная область и пример построения линий тока.

Из полученных результатов видно, что из-за более раннего прорыва воды (в момент времени 3000 дней) скорость закачки воды превышает результат, полученный на пятиточечном и девятиточечном разностных шаблонах (3500 дней). Это связано с тем, что фронт вытеснения направлен в диагональном направлении, поэтому при расчете на пятиточечном шаблоне 
фронту вытеснения приходится преодолевать больший путь, так как перетоки между ячейками в диагональном направлении отсутствуют. В то же время использование девятиточечного разностного шаблона значительно не меняет результат моделирования, так как доля потока, проходящего в диагональном направлении в 2 раза меньше доли потока в горизонтальном направлении. Кроме того, полученное методом линий тока распределение температуры физически достоверно (рис.6), а время, затрачиваемое на расчет, до 10 раз меньше времени расчета на пятиточечном разностном шаблоне.

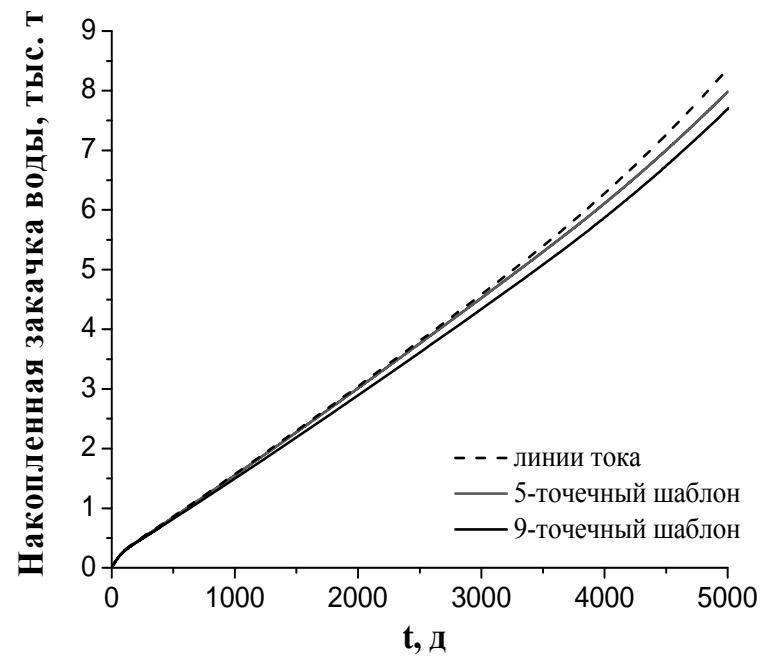

(a)

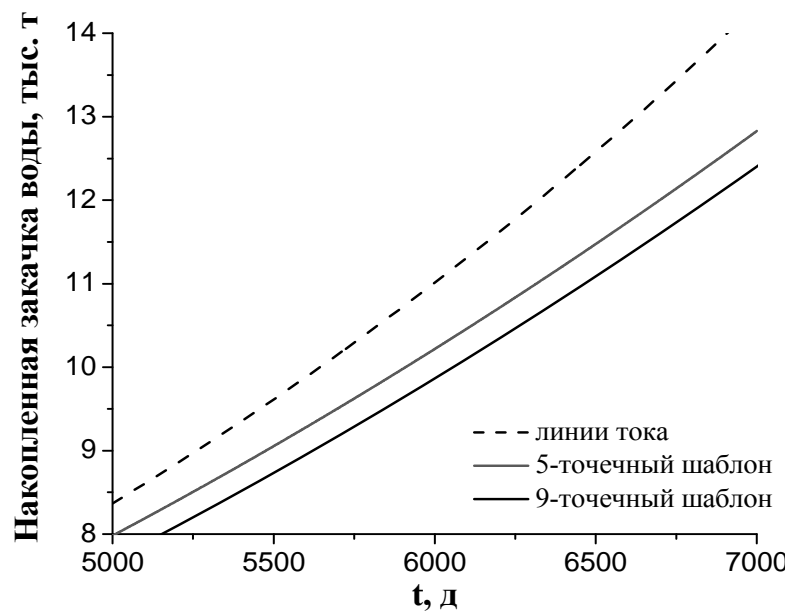

(б)

Рис.4. Зависимость накопленной закачки воды от времени при моделировании методом линий тока и методом конечных объемов: а - до 5000 дн.; б - с 5000 до 7000 дн.

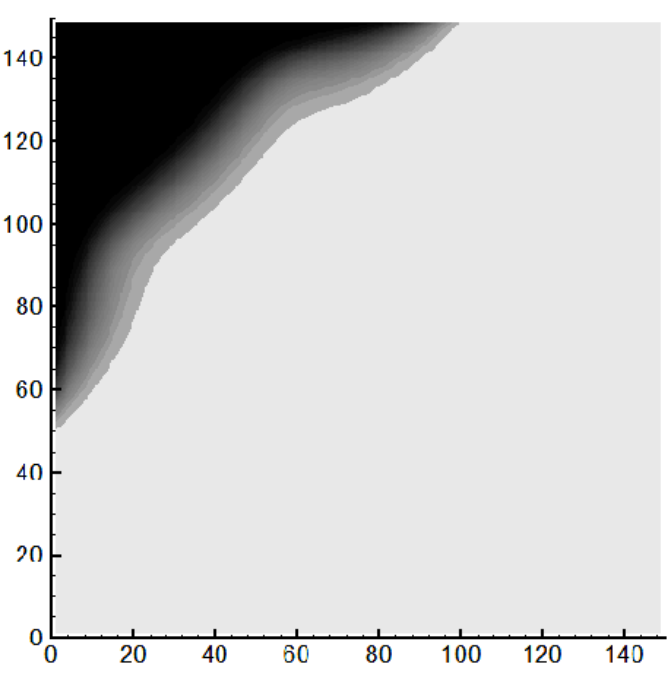

(a)

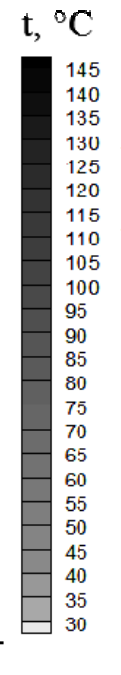

$\begin{array}{ll} & \\ 5 & 140 \\ 0 & \\ 5 & \\ 0 & 120 \\ 5 & \\ 0 & \\ 5 & \\ 0 & 100 \\ 5 & \\ 0 & \\ & 8 \\ & \\ & \\ & \end{array}$

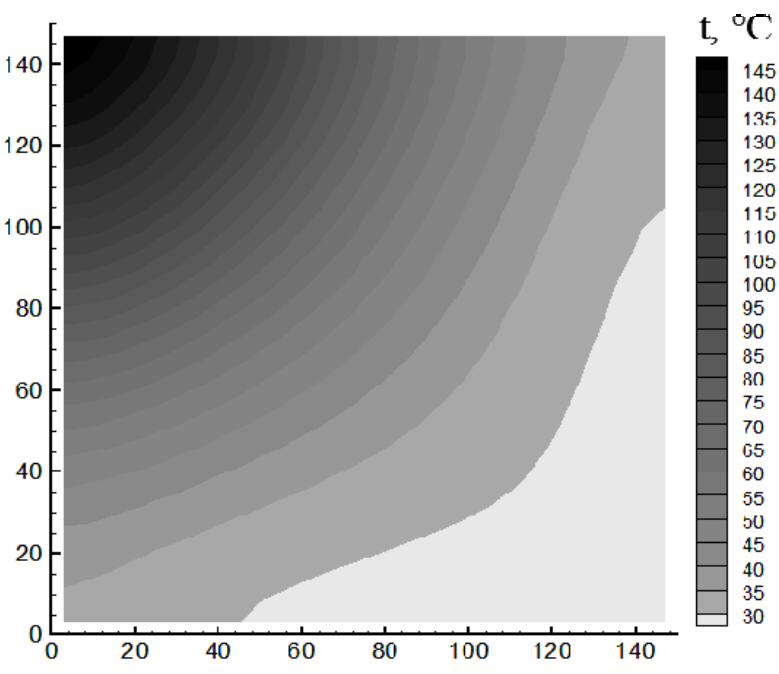

(б)

Рис.5. Поле температуры, полученное в результате моделирования вытеснения нефти ( $а$ - на пятиточечном разностном шаблоне; б - методом линий тока).

Помимо сравнения с результатами расчетов конечно-объемными методами в [17] было проведено исследование сходимости метода при изменении количества линий тока, различном числе ячеек на линиях тока, а также 
с различным соотношением между временем расчета на двумерной сетке и на линиях тока. В большинстве расчетов результаты слабо зависели от изменения указанных параметров. Исключения составил расчет с малым числом линий тока и грубой сеткой на линиях тока. В первом случае - погрешность была связана с тем, что линия тока не совпадала с осью ассоциированной трубки тока, во втором случае - с ошибкой интерполяции.

Таким образом, реализация метода линий тока позволяет проводить эффективные расчеты неизотермической фильтрации.

\section{5. Выводы}

В работе предложен новый метод линий тока, который показал высокую эффективность при решении задач неизотермической нелинейной фильтрации многокомпонентной смеси. Благодаря решению полной системы уравнений как на глобальной сетке, так и линиях тока, учтена зависимость закона фильтрации от температуры, что позволяет точнее определять геометрию линий тока и, таким образом, распределение неизвестных.

Для разработанного метода предложен алгоритм учета теплопроводности, основанный на расщеплении по физическим процессам, а также критерий остановки расчетов на линиях тока для последующего решения задачи на двумерной сетке.

Разработанные численные алгоритмы были реализованы в программном комплексе, который прошел верификацию и продемонстрировал свою эффективность по сравнению с традиционными конечно-объемными методами. Кроме того, разработанная программа может быть применена для практических расчетов при разработке месторождений вязкопластичных нефтей и интерпретации результатов испытаний скважин.

\section{СПИСОК ЛИТЕРАТУРЫ}

1. W. Xiong, Q. Lei, S. Gao, Z. Hu, H. Xue. Pseudo threshold pressure gradient to flow for low permeability reservoirs // Petroleum Exploration and Development, 2009, v.36, p.232-236.

2. V.T. Beraldo, M.J. Blunt, D.J. Schiozer. Compressible streamline-based simulation with changes in oil composition // SPE Reservoir Evaluation and Engineering, 2009, v.12, p.963-973.

3. B.T. Mallison. Streamline-Based Simulation of Two-phase, Multicomponent Flow in Porous Media: PhD dissertation. - Stanford University, 2004, 104 p.

4. M.R. Thiele. Modeling Multiphase Flow in Heterogeneous Media Using Streamtubes: PhD dissertation. - Stanford University, 1994, 203 p.

5. P. Usman. Development of Streamline-Based Simulators for Evaluation of Heavy Oil Recovery: PhD dissertation. - Waseda University, Tokyo, 2007, 161 p. 
6. Z. Zhu. Efficient simulation of thermal enhances oil recovery processes: $\mathrm{PhD}$ dissertation. - Stanford University, 2011, $215 \mathrm{p}$.

7. H. Cheng, I. Osako, A. Datta-Gupta, M. King. A rigorous compressible streamline formulation for two- and three-phase black-oil simulation // SPE Reservoir Evaluation and Engineering, 2006, v.11, p.407-417.

8. Z.X. Pang, H.Q. Liu. The transient method and experimental study on threshold pressure gradient of heavy oil in porous media // Petroleum Engineering Journal, 2012, v.5, p.7-13.

9. А.Х. Мирзаджанзаде. Вопросы гидродинамики вязкопластичных и вязких жидкостей в применении к нефтедобыче. - Баку: Азернефтнешр, 1959, 409 с.

A.Kh. Mirzadzhanzade. Voprosy gidrodinamiki viazkoplastichnyh i viazkikh zhidkostei v primenenii k neftedobyche. - Baku: Azerneftnashr, 1959, $409 \mathrm{~s}$.

10. Advanced Process and Thermal Reservoir Simulator; CMG STARS, Version 2009 [Text] : Computer Modelling Group Ltd. - Calgary, AB, Canada, 2009, 1120 p.

11. Eclipse. 2009. Eclipse Version 2009 Software Manual. Schlumberger Ltd., 2003, 1068 p.

12. Д.Ф. Сиковский. Методы вычислительной теплопередачи: Учебное пособие. - Новосибирск: Новосибирский государственный университет, 2011, 121 с.;

D.F. Sikovskiy. Metody vychislitelnoi teploperedachi: Uchebnoe posobie. - Novosibirsk: Novosibirsk state University, 2011, $121 \mathrm{~s}$.

13. А.И. Брусиловский. Фазовые превращения при разработке месторождений нефти и газа. - М.: «Грааль», 2002, 575 с.;

A.I. Brusilovsky. Phase transformations in the development of oil and gas. - M.: The Grail, 2002, $572 \mathrm{p}$.

14. P.K.W. Vinsome, J. Westerveld. A simple method for predicting cap and base rock heat losses in thermal reservoir simulators // Journal of Canadian Petroleum Technology, 1980, v.19, p.87-90.

15. D.W. Pollock. Semianalytical computation of path lines for finite-difference models // Ground Water, 1988, v.26. p.743-750.

16. A. Bordbar, S. Faroughi, S.A. Faroughi. A Pseudo-TOF Based Streamline Tracing For Streamline Simulation Method in Heterogeneous Hydrocarbon Reservoirs // American Journal of Engineering Research, 2018, v.7, p.23-31.

17. Я.В. Невмержицкий. Применение метода линий тока для ускорения расчетов неизотермической нелинейной фильтрации // Компьютерные исследования и моделирование, 2018, т.10, №5, с.709-728;

Ya.V. Nevmerzhitskiy. Primenenie metoda linii toka dlia uskoreniia raschetov neizotermicheskoi nelineinoi filtratsii // Kompiuternye issledovaniia i modelirovanie, 2018, t.10, №5, s. 709-728.

Поступила в редакцию 27.06.2019

После доработки 27.06.2019

Принята к публикации 25.11.2019 